\title{
The Impact of Fragmented Habitat's Size and Shape on Populations with Allee Effect
}

\author{
W. G. Alharbi, S. V. Petrovskii * \\ Department of Mathematics, University of Leicester, University Road, Leicester LE1 7RH, UK
}

\begin{abstract}
This study aims to explore the ways in which population dynamics are affected by the shape and size of fragmented habitats. Habitat fragmentation has become a key concern in ecology over the past 20 years as it is thought to increase the threat of extinction for a number of plant and animal species; particularly those close to the fragment edge. In this study, we consider this issue using mathematical modelling and computer simulations in several domains of various shape and with different strength of the Allee effect. A two-dimensional reaction-diffusion equation (taking the Allee effect into account) is used as a model. Extensive simulations are performed in order to determine how the boundaries impact the population persistence. Our results indicate the following: (i) for domains of simple shape (e.g. rectangle), the effect of the critical patch size (amplified by the Allee effect) is similar to what is observed in 1D space, in particular, the likelihood of population survival is determined by the interplay between the domain size and thee strength of the Allee effect; (ii) in domains of complicated shape, for the population to survive, the domain area needs to be larger than the area of the corresponding rectangle. Hence, it can be concluded that domain size and shape both have crucial effect on population survival.
\end{abstract}

Keywords and phrases: extinction, fragment size, habitat fragmentation, edge effects

Mathematics Subject Classification: 92D40, 35B36, 35Q92, 37N25

\section{Introduction}

Due to ill-planned human action and unsustainable exploitation of resources, fragmentation of ecological habitats is becoming more common and has become a process that can negatively affect the survival rates of many species $[12,17,34,37]$. Consequently, for the past two decades, the study of population dynamics with regards to fragmented habitats has become an issue of considerable importance for conservationists and other parties interested in certain species survival $[4,12]$. The ecological effects of fragmentation have received increasing attention in many recent studies focusing on the issue of habitat fragmentation and related aspects (e.g. formation of habitat boundary ecosystem) [12,13,34,36]. Mathematical models confirming that small and fragmented populations are more likely to go extinct have been developed and used as a result thereof $[8,16,24,25,35]$.

${ }^{*}$ Corresponding author. E-mail: sp237@le.ac.uk 
The effects of the fragmentation processes on habitats can be observed in two ways, either in the shortterm (including modifications of habitat shapes, changes in population sizes, and the emerging structures at the edges of the fragments) or in the long-term (including population genetic transformations and extinction of species that have either low growth rate or a complex life cycles) [2]. Furthermore, fragmentation can have either a direct or indirect impact on different types of plant and animal species. Generally speaking, plants and animals react in one of three ways to habitat fragmentation: they can respond positively (e.g. by an increase in the population size of pioneer species of plants, modifications in species composition, and changes in distribution and biotic interactions), negatively (such as by experiencing a decrease in the population size, as it happens for many species), or neutrally (meaning that fragmentation had no clear effect on them). This latter example can be seen in the case of the fragmentation of the habitat of Los Tuxtlas, Mexico, where a continuous forest covering $1000 \mathrm{~m}^{2}$ showed no difference in the population size of a particular plant species both prior to and after fragmentation [2]. As a rule, the effect of habitat fragmentation is felt strongest by plant species, especially whose seeds are disseminated by animals.

In the ecosystems science, a major area of study has been the effect of fragmentation on population dynamics. A wide range of effects has been discovered, revealing the influence of various factors including (1) fragment size, (2) edge effects, (3) fragment shape, (4) isolation, and (5) the characteristics of the environment "matrix" surrounding the fragments (e.g. native vegetation type such as deforested areas, cattle pasture, agricultural crops, urban areas, etc.) $[2,4,5,11,12]$. However, the dynamics have in some cases proven difficult for biologists to study and evaluate, for various reasons including (i) the response time of populations to fragmentation, (ii) the biogeographical position of the species under study, and (iii) synergism between different processes (e.g. between fragmentation and harvesting, or fragmentation and climatic change). For these reasons, mathematical modelling is used extensively. In this paper, we use mathematical modelling and simulation to study the effects of fragmentation on population dynamics as a function of (1) fragment size, (2) edge effects, and (3) fragment shape.

Edge Effects. One important outcome of habitat fragmentation is the emergence of the habitat edges. Ewers and Didham [11] explained this by referring to those sections of a fragment that have been modified by outside agents and, therefore, implicitly experienced edge effects. These effects manifest themselves in the manner in which two neighbouring ecosystems interact through the common boundary [13]. As noted by Ewers and Didham [11], habitat fragmentation increases the chances of changes to occur. This is seen most prominently closer to the edge. Habitat edges play a key role not only in regulating the flow of organisms but also in permitting the invasion of external influences, such as fire and non-native species. Closeness to the fragment edge has an impact on the persistence and survival of animal and plant species as well as on their interactions. The survival and persistence of animal and plant species and their subsequent interactions are also influenced when they are nearer to the fragment edge [2].

Fragment Size. Fragment size has been identified by numerous researchers as the most important spatial feature that has a significant effect on the biodiversity in a fragmented landscape $[4,11,23]$. Expansive areas are generally good for the survival and proliferation of plants and animals. As a result thereof, the extent to which a species is susceptible to potential extinction increases along with a decrease in the fragment size.

Fragment Shape. Ecological interactions are known to go differently in areas adjacent to the habitat border and in the core areas [5,27]. On the other hand, for a given area of the habitat, domains of complex shape tend to have longer boundary and/or smaller core areas. One can conjecture that the population dynamics (in particular, the likelihood of population survival) should be dependent on the habitat shape. Indeed, the connection between the shape of the fragment and the richness of the population species living therein was noticed in some earlier work [30], although the measures to determine the importance of the shape of the fragment remained largely speculative.

In order to predict the changes in population dynamics or community structure resulting from a change in landscape structure, it is necessary to understand the interrelationship between the essential components [9]. The interplay between the fragment size and fragment shape has a decisive influence 
on the area of a habitat fragment that is likely to feel the impact of the edge effects [11]. Moreover, as was mentioned above, whether animal and plant species will survive or not is often determined by the proximity to fragment edges [2]. On the other hand, it was also also observed that, under certain circumstances, plant and animal populations may be favourably influenced by habitat fragments of large size and with an irregular shape [2]. This favourable influence of complex shapes on species of plants and animals may be explained in terms of the fact that, by comparison to more compact fragments, they permit colonisation by different populations with greater frequency. Ewers and Didham [11] clarified that intensified colonization is made possible by the higher number of edges possessed by fragments with a complex shape.

The impact of fragmentation on population dynamics is the main focus of the present study, with special attention being given to the effects of fragment shape and size. The size and shape of a domain have a significant influence on the properties and characteristics displayed by the population dynamics [29]. To gain an understanding of this influence, different fragment shapes will be analysed, with a particular focus on the existence of critical patch size effects, taking into account three different normalisations of the population growth rates [28]; see Eqs. (2.3-2.5) in Section 2. Since the critical patch size of a population is the minimum size of habitat necessary for population persistence, its estimation is clearly crucial for understanding population dynamics. The earliest investigation of critical patch size was performed by Skellam [26] and Kierstead \& Slobodkin [19]. They derived the critical size for continuous-time models displaying exponential growth and simple diffusion, i.e. for the following equation:

$$
\frac{\partial u}{\partial t}=\alpha u+D \nabla^{2} u
$$

where $\alpha$ is the linear growth rate and $D$ is the coefficient of diffusion. Having considered Eq. (1.1) in a one-dimensional habitat with length $L$, they obtained the following formula:

$$
L_{S r}=\pi \sqrt{\frac{D}{\alpha}} .
$$

The species survives if $L>L_{C r}$, but will be at risk of becoming extinct if $L<L_{C r}$.

In the case of a two-dimensional domain square domain $L \times L$, the condition of population survival is given by:

$$
L>L_{S r}=\pi \sqrt{\frac{2 D}{\alpha}},
$$

which obviously is a generalization of (1.2). Formula (1.3) is easy to derive (by considering the eigenvalues of the problem) and it is apparently far too simple in the context of real landscapes. A shape of greater complexity is likely to be more realistic. Based on this observation, the following point can be deduced: the variety of shapes manifested by different habitats can result in wide variations in critical patch size and, consequently, wide variations in the efficiency of population survival.

In this study we will analyse the effect of three fragment shapes, namely, H-shaped, cross-shaped and square. A numerical solution of the two-dimensional reaction-diffusion equation (taking the Allee effect into account) will be employed to analyse the critical sizes of these habitat shapes for a single species. We will discuss (a) possible explanations for the critical habitat sizes of these shapes using different normalisations of the population growth rates, and (b) the effect of shape complexity in determining whether a population persists or goes extinct.

\section{Mathematical Model}

Our model is given by the following reaction-diffusion equation [10,21,31,32, 39]:

$$
\frac{\partial u}{\partial t}=f(u)+D \nabla^{2} u
$$


where $u$ is the population density, $D$ is the diffusion coefficient, $\nabla^{2}$ is the Laplacian defined as $\frac{\partial^{2}}{\partial x^{2}}$ for the one-dimensional (1D) case and $\left[\frac{\partial^{2}}{\partial x^{2}}+\frac{\partial^{2}}{\partial y^{2}}\right]$ for the two-dimensional (2D) case, and $f(u)$ is the population growth rate.

Assuming that the growth rate is dampened by the Allee effect $[28,33]$ (as indeed is often the case $[6,8]$ ), the per capita growth rate is dome-shaped and hence can be approximated with a square polynomial, which results in the cubic-polynomial expression for the growth rate $f$ :

$$
f(u)=\gamma u(1-u)(u-\beta),
$$

where $\gamma$ is a coefficient which can be regarded as the characteristic growth rate. Note that the carrying capacity is scaled to one. Parameter $\beta$ is known as the Allee threshold or threshold density and can be used to estimate how strong the Allee effect is. The Allee effect is considered weak when $-1<\beta<0$ and is considered strong when $0<\beta$ [28]. Here the values $0.5<\beta<1$ are sensible but not interesting because for these values a sufficiently large perturbation of the upper steady state $u=1$ would normally lead to the population extinction in the spatial system $(2.1)$.

Note that every different choice of parameters $\gamma$ and $\beta$ corresponds, generally speaking, to a different population. In order to study the effect of habitat's size and shape on different populations, one should be able to compare those different populations in a sensible way. Sensible comparison between two populations defined by two arbitrary parameter pairs, say $\left(\gamma_{1}, \beta_{1}\right)$ and $\left(\gamma_{2}, \beta_{2}\right)$ is difficult if possible at all. Indeed, different $\gamma$ and $\beta$ result in the growth rate of a different shape, and so what? In order to resolve this generic difficulty, Lewis \& Kareiva [28] suggested to fix a certain feature of the population growth so that variation of the parameters would leave it unchanged. (For instance, such a feature can be the maximum growth, i.e. the maximum value of function $f(u)$ for $0<u<1$.) Introducing this constraint would introduce a relation between $\gamma$ and $\beta$, hence rendering only one of the being independent (say, $\beta$ ) and the other one becoming its function, say $\gamma(\beta)$. Following [28], we therefore consider three different options:

1. The simplest constraint is to assume that $\gamma$ always has a fixed value, thus being just a constant number:

$$
\gamma=\text { constant }
$$

For the sake of simplicity, below we consider $\gamma=1$.

2. In the case that the growth rate $f(u)$ as given by Eq. (2.2) is constrained to attain a fixed maximum value, then it is straightforward to derive the following expression:

$$
\gamma=27 /\left(2\left(\left((1+\beta)^{2}-9 \beta / 2\right)(1+\beta)+\left((1+\beta)^{2}-3 \beta\right)^{\frac{3}{2}}\right)\right) .
$$

3. In the case the maximum per capita growth rate is assumed to have a constant value, then it is readily seen that

$$
\gamma=4 /(1-\beta)^{2}
$$

In the next section, in order to reveal the effect of the size and shape, the $2 \mathrm{D}$ dynamics of the spatial system (2.1) will be considered in the domain of three different shapes (H-shaped, cross-shaped, and a simple square) subject to the different normalizations (2.3-2.5).

\section{Results of computer simulations}

In this section, we will present the results of the numerical simulations. Eq. (2.1) is solved numerically by finite differences using the explicit scheme. The values of the grid steps $\delta x$ and $\delta t$ are chosen to be sufficiently small in order to avoid numerical artifacts. Furthermore, before proceeding to the numerical simulations, the equations are required to be supplemented with boundary and initial conditions. At the boundary $\Gamma$ of the domain, we use the 'zero-function' Dirichlet condition, i.e. $u(\mathbf{r}, t)=0$ for $\mathbf{r} \in \Gamma$. 
For the initial values, we use $u(x, y, 0)=u_{0}=$ constant. The growth rate was chosen in line with Eqs. (2.3-2.5). The diffusivity is fixed as $D=1$.

We first consider a special case where the growth rate is linear, i.e. where the dynamics is described by Eq. (1.1). For the square-shaped domain, the critical size is given by Eq. (1.3). As the next level of geometrical complexity, we consider Eq. (1.1) in a cross-shaped domain (see Fig. 1) which is obtained from the square-shaped $L \times L$ domain by removing equal $l \times l$ squares from its every corner (where, obviously, $0<l<0.5 L$ ). Numerical simulations show that there indeed exists the critical size that tends to decrease with the an increase in the growth rate $\alpha$; see Figs. 2 and 3.

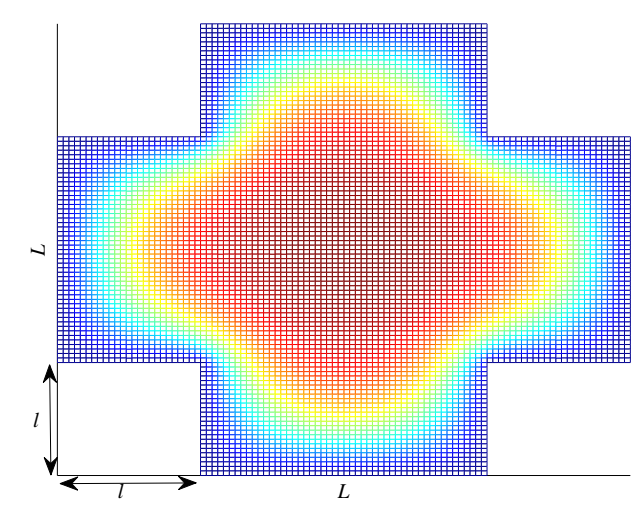

Figure 1: A sketch of the solution of the reaction-diffusion equation in the 2D cross-shaped domain.

We then consider the specific question whether it may be possible to match the critical size of the cross-shaped domain with that of the square where the results is known; see Eq. (1.3). Intuitively, the matching factor should take into account the area removed at the corners of the original square, which leads to the following heuristic relation between the critical size of the cross-shaped domain and that of the square:

$$
L_{C r}=L_{S r}\left[\frac{1}{1-4 \zeta^{2}}\right],
$$

where $L_{S r}$ is the critical size of the square (as is given by Eq. 1.3), $L_{C r}$ represents the critical size of the cross-shaped domain and $\zeta=l / L$, so that $0<\zeta<0.5$. Surprisingly, the simple formula (3.1) gives very good result when $\zeta$ is not large; see Fig. 2. The agreement is becoming somewhat worse when $\zeta$ approaches its maximum possible value 0.5 . We mention here that the matching relation (3.1) can be made more accurate by introducing the correcting term, say $\rho(\zeta)$ :

$$
L_{C r}=L_{S r}\left[\frac{1}{1-4 \zeta^{2}}+\rho(\zeta)\right] .
$$

In particular, we have found that the following choice of function $\rho(\zeta)$ :

$$
\rho(\zeta)=-584 \zeta(-0.5+\zeta)\left(-0.004+0.074 \zeta-0.528 \zeta^{2}+\zeta^{3}\right)
$$

makes the matching relation (3.2) almost precise; see Fig. 3. However, we admit that the choice of the correcting term as (3.3) is purely technical (having been obtained by best-fitting the simulation data with polynomials of a certain order) and is difficult to explain in heuristic terms.

The linear population growth considered above, although providing a good approximation in case the population density is not large and there is no strong Allee effect, is not realistic if considered in a more 


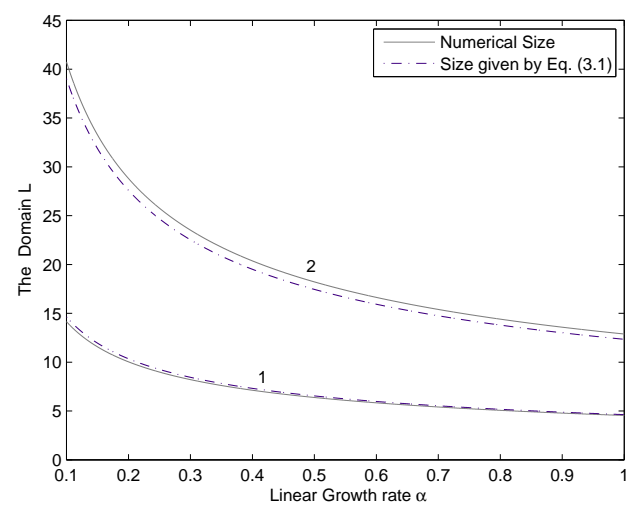

Figure 2: Critical size of the cross-shaped domain obtained at different values of linear growth rate $\alpha$. The solid curves show the critical size as given by the numerical simulations and the dashed-and-dotted curves show the critical size as given by Eq. (3.1) for (1) $\zeta=0.1$ and (2) $\zeta=0.4$.

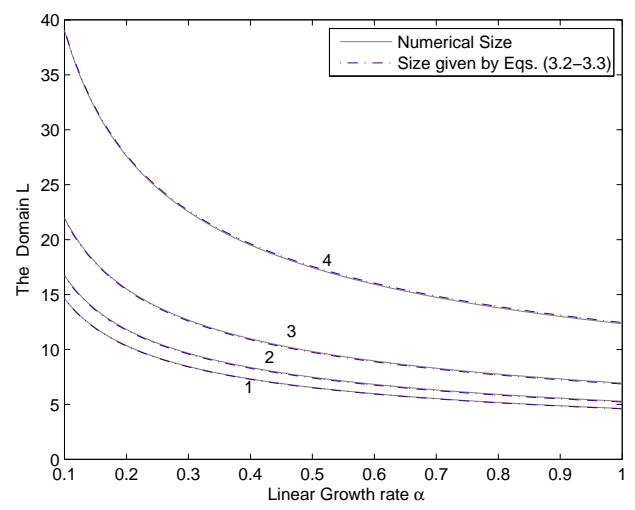

Figure 3: Critical size of cross-shaped domain obtained at different values of linear growth rate $\alpha$. The solid curves show the critical size as given by the numerical simulations and the dashed-and-dotted curves show the critical size as given by Eqs. (3.2-3.3) for $(1) \zeta=0.1,(2) \zeta=0.2,(3) \zeta=0.3$ and (4) $\zeta=0.4$.

general case. We therefore proceed now to a more general model (2.1) with the growth rate given by (2.2) with one of the scaling relations (2.3-2.5). In this case, there is no analytical solution to the critical size problem and the system is studied through extensive numerical simulations. The results obtained for the two different shapes (i.e. square and cross) and the three different choices for $\gamma(\beta)$ are shown in Figs. 4-6. The critical relation between $L$ and $\beta$ is shown by the solid curve (obtained numerically); the parameters from above the curve correspond to population survival, the parameters from below the curve correspond to population extinction. We therefore observe that the general system (2.1-2.2) exhibits essentially the same tendency with regard to the domain shape as the linear system (1.1): for the same value of $L$ and $\beta$, the critical size of the cross-shaped domain is always larger than the critical size of the square-shaped domain. This clearly manifests the importance of the domain shape for population survival. We also observe that the critical size tends to increase with an increase in the strength of the Allee effect (quantified by parameter $\beta$ ). Interestingly, the rate of increase appears to depend on the choice of the scaling relation for $\gamma$. In particular, when $\gamma$ is scaled as (2.5) (i.e. to keep the same value of the maximum per capita growth), the critical size is approximately constant for $\beta<0$ where the Allee effect is weak. 
(a)

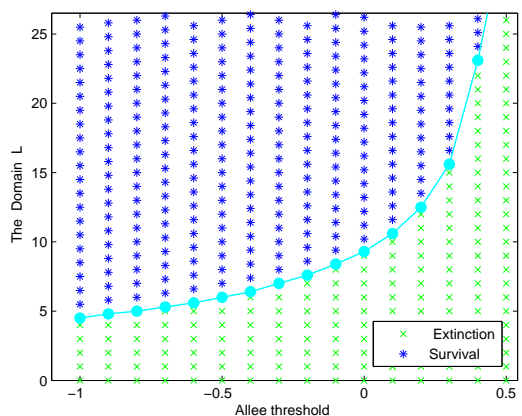

(b)

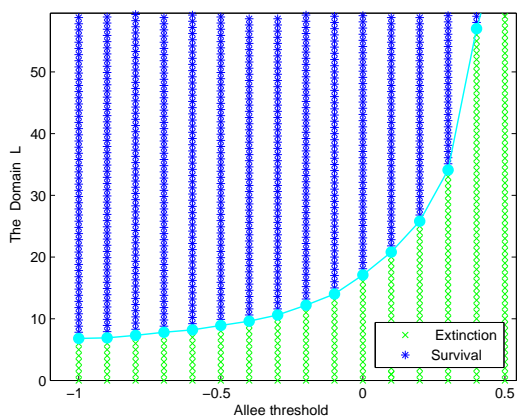

Figure 4: The structure of the parameter plane $(\beta, L)$. The parameters from above the solid curve correspond to population survival and the parameters from below the curve correspond to extinction. The population growth rate is scaled according to Eq. (2.3). (a) Square shaped domain, (b) cross-shaped domain with $(l / L=0.3)$.

(a)

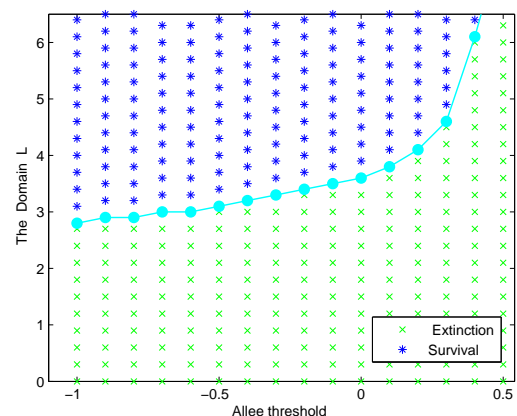

(b)

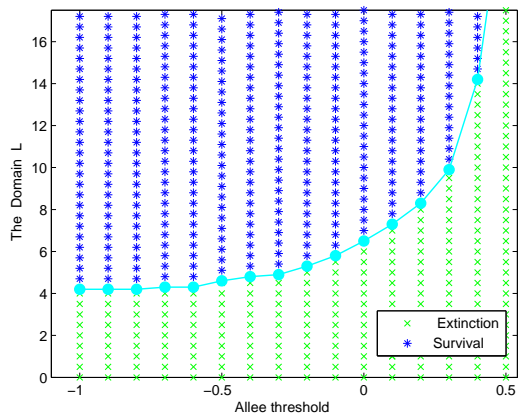

Figure 5: The structure of the parameter plane $(\beta, L)$ obtained when the population growth rate is scaled according to Eq. (2.4). (a) Square shaped domain, (b) cross-shaped domain with $(l / L=0.3)$.

(a)

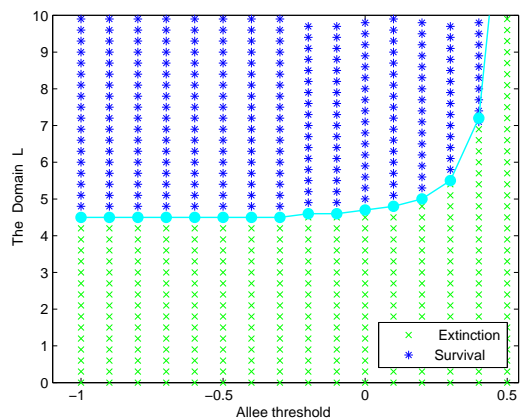

(b)

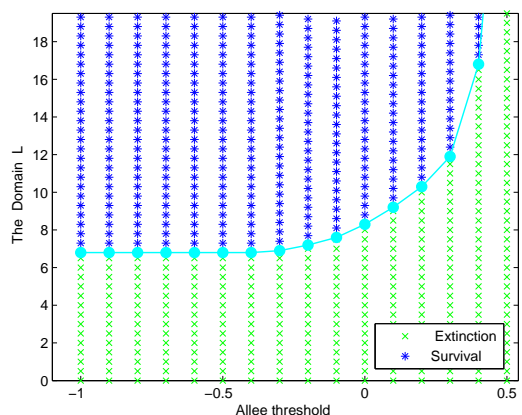

Figure 6: The structure of the parameter plane $(\beta, L)$ obtained when the population growth rate is scaled according to Eq. (2.5). (a) Square shaped domain, (b) cross-shaped domain with $(l / L=0.3)$.

In order to further reveal the effects of the domain geometry on the population dynamics, now we are going to consider the domain of a different shape. For convenience, we refer to it as the 'H-shaped' domain; see Fig. 7. There are therefore two large sub-domains (Habitat 1 and Habitat 2) and the narrow passage connecting them. Our choice of this shape is motivated by the biological observations pointing 
out at the importance of corridors for the population dynamics [2]. We will consider the overall size of the domain to be large enough $(L=100)$, so that the size of Habitats 1 and 2 are overcritical; hence, the population would not be endangered in either of them. Our aim is to reveal the effect of the passage. We choose the initial conditions differently from the above, namely $u_{0}=1$ in Habitat 1 and $u_{0}=0$ in the rest of the domain, i.e. in Habitat 2 and the passage. As well as above, the Dirichlet boundary conditions $u(\mathbf{r}, t)=0$ are used at the domain boundary. Our primary interest here is to reveal how the parameters of the passage, i.e. its length and width, can affect the population spread from Habitat 1 to Habitat 2.

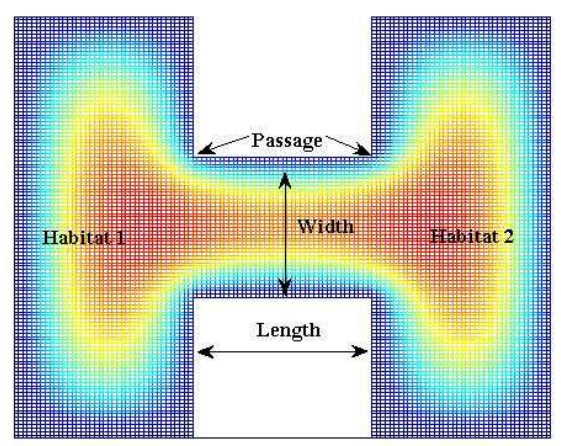

Figure 7: A sketch of the solution of the 2D reaction-diffusion equation affected by the domain shape, in particular, by the parameters (i.e. width and length) of the passage between the two large sub-domains.

(a)

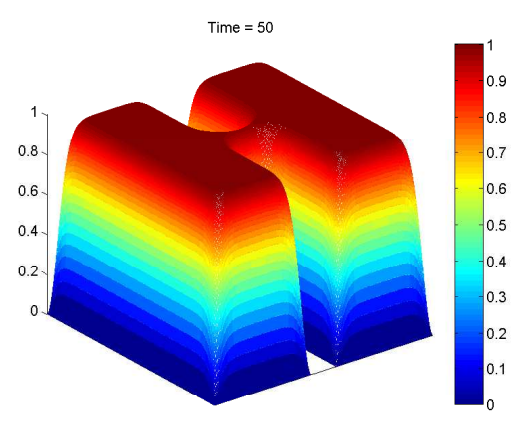

(b)

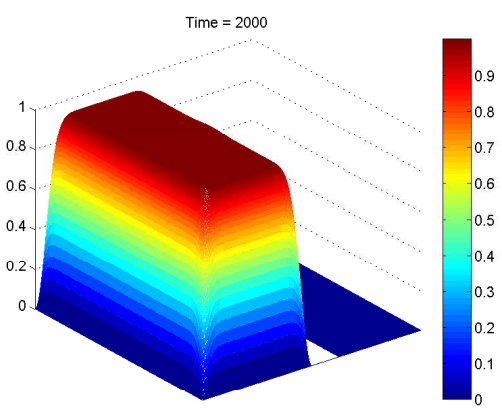

Figure 8: The simulation results showing the spread of the population to Habitat 2 in case of (a) shorter passage with (length, width) $=(4.6,7)$ and $(\mathrm{b})$ population blocking for a longer passage with (length, width $)=(5,7)$. Other parameters are $D=1$ and $\beta=0.1$, and $\gamma(\beta)$ is given by Eq. (2.5).

Figure 8 shows some typical simulation results. Having fixed the passage width as 7 , we observe that the population spills over to Habitat 2 for the passage length being 4.6 but is blocked by the passage when its length is equal to 5 . The existence of critical parameters of the passage is thus demonstrated. Intuitively, we can therefore expect that the population is going to be blocked for a sufficiently narrow or sufficiently long passage but could spill over to Habitat 2 otherwise. This expectation is generally confirmed by our simulation results; see Fig. 9. Additionally, we observe that there exists a critical value $w_{c r}$ of the passage width, $w_{c r} \approx 7.8, w_{c r} \approx 3.0$ and $w_{c r} \approx 3.6$ for Fig. $9 \mathrm{a}, \mathrm{b}$ and $\mathrm{c}$, respectively. In case 
the width is larger than $w_{c r}$, the population cannot be blocked whatever is the length of the passage. Note that the critical width appears to be about 2.5 times larger in case of the scaling (2.3) (Fig. 9a) compared to the scaling (2.4) (Fig. 9b).

(a)

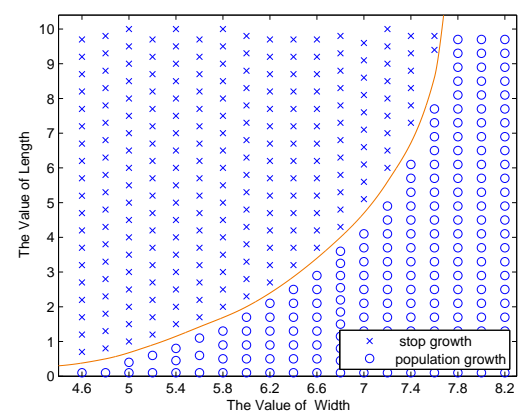

(b)

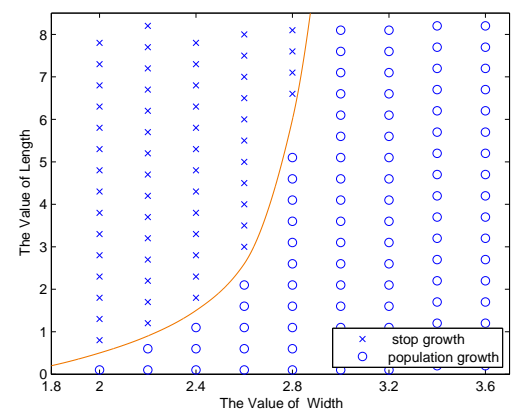

(c)

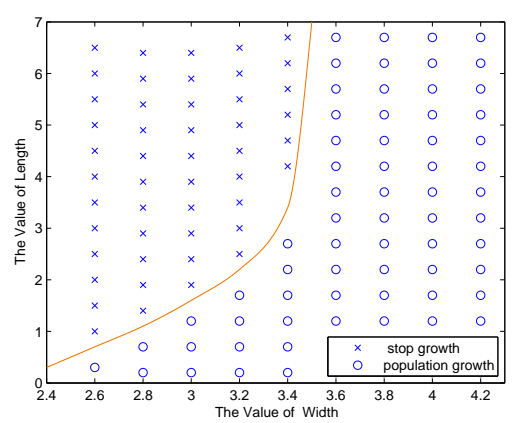

Figure 9: Parameter plane (width, length) of the H-shaped domain (see Fig 7). The solid curve shows the critical relationship between the width and length of the passage. For the parameters from below the line, the population invades through the passage. For the parameters from above the line, the passage is blocked. Here (a), (b) and (c) correspond to the the population growth rate $\gamma$ scaled according to Eq. (2.3), Eq. (2.4), and Eq. (2.5), respectively.

\section{Discussion and concluding remarks}

In this paper, we have considered the effect of the domain size and shape on the population dynamics, in particular, on the population survival in case the overall size of the habitat is small. We have addressed this problem theoretically using the single species model described by a scalar reaction-diffusion equation. Relevant properties of the solution such as either $u(\mathbf{r}, t) \rightarrow 0$ for $t \rightarrow \infty$ uniformly over the domain (extinction) or otherwise (survival) were revealed in extensive computer simulations subject to domain's shape and size, the strength of the Allee effect and different scaling (normalization) of the growth rate. Having analyzed the simulation results in the special case of the linear growth rate, we found a simple formula to match the critical size of the cross-shaped domain to that of the square, thus making the corresponding problem analytically tractable.

Having considered three different normalizations for the population growth rate $\gamma$, see Eqs. (2.3-2.5), we have found that the features of the critical size dependence on the strength of the Allee effect are sensitive to the way how different populations are compared. In particular, in the square-shaped domain, in the range $-1<\beta<0$ (i.e. when the Allee effect is weak) the critical size increases almost linearly in 
case of normalization (2.4) but remains approximately constant in case of normalization (2.5), cf. Figs. 5a and $6 a$.

Sensitivity of the critical patch size to the growth rate normalization is a rather counterintuitive result that can have various implications. Firstly, in the context of mathematical modelling, this evokes the concept of structural stability. Some population dynamics models are known to show high sensitivity to the details of the functional response(s), so that the bifurcation value of the controlling parameter (e.g. the carrying capacity) can differ by an order of magnitude $[1,14,15]$. Interestingly, in our model, the functional form of the population growth is exactly the same for all three normalizations, only the constraint on the parameter values is somewhat different, and yet the obtained critical patch size can differ more than twice (e.g. compare Figs. $4 \mathrm{a}$ and 5a) and responds differently to the change in the threshold density $\beta$ (cf. the different shape of the corresponding curves in Figs. 5 and 6). It suggests that, when building even a simple single species model, one has to be careful about the biological justification of the details.

Secondly, different value of the critical size and its different dependence on $\beta$ may also indicate the existence of certain evolutionary strategies in response to habitat fragmentation. Indeed, according to the meaning of normalizations (2.4) and (2.5), such evolution would be going on while trying to preserve, respectively, either the maximum growth rate or the maximum per capita growth rate. However, in case of (2.5), the critical patch size appears to be almost two times larger than in case of (2.4). Similarly, a hypothetical species with the strategy according to (2.4) would show a much better capability to spread between different habitats in a poorly connected landscape than a species with the strategy (2.5); cf. Figs. $9 \mathrm{~b}$ and 9c. It suggests that the species employing the strategy to maintain its maximum growth rate (rather than per capita growth rate) would likely have more chances to survive in a complex or heavily fragmented environment.

As was previously discussed, the size and shape of the habitat both have a significant influence on the population dynamics, in particular on its survival. We have shown that, although the size of a domain is important, in reality it is actually the interplay between the size and shape of a given domain that determines the survival of its population. One important finding of our study is that the critical size of the cross-shaped domain is always larger than the corresponding critical size of the square-shaped domain. It suggests that the likelihood of the population survival is higher in fragmented habitats of simple shapes. This conclusion agrees well with the empirical observation that the probability of population persistence in fragments with complex shapes can sometimes be reduced $[3,20]$. Similarly, the fraction of the habitat area that is affected by the edge effects is known to be determined by the connection between the shape and the size of the habitat [2]. Thus, our study provides a relevant theoretical framework for the interpretation of empirical observations on the population dynamics on fragmented habitats that can be used in further studies on this important research topic.

\section{References}

[1] M.W. Adamson, and A. Y. Morozov. When can we trust our model predictions? Unearthing structural sensitivity in biological systems. Proc. R. Soc. A. The Royal Society, 469 (2012), 20120500.

[2] J. Benitez-Malvido, V. Arroyo-Rodriguez. Habitat fragmentation, edge effects and biological corridors in tropical ecosystems. Encyclopaedia of Life Support Systems (EOLSS), Oxford: Eolss Publishers, (2008), 1-11.

[3] M. Bevers, C. H. Flather. Numerically exploring habitat fragmentation effects on populations using cell-based coupled map lattices. Theor. Popul. Biol., 55(1) (1999), 61-76.

[4] P. Cheptou, V. Avendano, G. Lyz. Pollination processes and the Allee effect in highly fragmented populations: consequences for the mating system in urban environments. New Phytol., 172(4) (2006), 774-783.

[5] S. K. Collinge. Ecological consequences of habitat fragmentation: implications for landscape architecture and planning. Landsc. Urban Plan., 36(1) (1996), 59-77.

[6] F. Courchamp, T. Clutton-Brock, B. Grenfell. Inverse density dependence and the Allee effect. Trends Ecol. Evol., 14(10) (1999), 405-410.

[7] J. Crank. The Mathematics of Diffusion. Oxford University Press, 1979.

[8] B. Dennis. Allee effects: population growth, critical density, and the chance of extinction. Nat. Resour. Model., 3(4) (1989), 481-538. 
[9] J. B. Dunning, B. J. Danielson, H. R. Pulliam. Ecological Processes that Affect Populations in Complex Landscapes. Oikos, 65(1) (1992), 169-175.

[10] L. Edelstein-Keshet. Mathematical Models in Biology. SIAM, 1988.

[11] R. M. Ewers, R. K. Didham. Confounding factors in the detection of species responses to habitat fragmentation. Biol. Rev., 81(01) (2006), 117-142.

[12] L. Fahrig. Effects of habitat fragmentation on biodiversity. Annu. Rev. Ecol. Evol. Syst., 34 (2003), $487-515$.

[13] J. Fischer, D.B. Lindenmayer. Landscape modification and habitat fragmentation: a synthesis. Glob. Ecol. Biogeogr., 16(3) (2007), 265-280.

[14] C. Flora, N. David, G. Mathias, A. Morozov, andP. Jean-Christophe. Structural sensitivity of biological models revisited. J. Theor. Biol., 283(1) (2011), 82-91.

[15] G.F. Fussmann, and B. Blasius. Community response to enrichment is highly sensitive to model structure. Biol. Lett., 1(1) (2005), 9-12.

[16] D. Goodman. Consideration of stochastic demography in the design and management of biological reserves. Nat. Resour. Model., 1 (1987a), 205-234.

[17] I. Hanksi, M. Gilpin. Metapopulation dynamics: brief history and conceptual domain. Biol. J. Linn. Soc., 42(1-2) (1991), 3-16.

[18] M. Jankovic, S. Petrovskii. Are time delays always destabilizing? Revisiting the role of time delays and the Allee effect. Theor. Ecol., 7(4) (2014), 335-349.

[19] H. Kierstead, L.B. Slobodkin. The size of water masses containing plankton blooms. J. mar. Res., 12(1) (1953), 141-147

[20] R. V. Kirk, M. A. Lewis. Edge permeability and population persistence in isolated habitat patches. Nat. Resour. Model., 12(1) (1999), 37-64.

[21] M. Kot. Elements of Mathematical Ecology. Cambridge University Press, 2001.

[22] W. Kunin, Y. Iwasa. Pollinator foraging strategies in mixed floral arrays: density effects and floral constancy. Theor. Popul. Biol., 49(2) (1996), 232-263.

[23] B. B. Lamont, P. G. Klinkhamer, E. Witkowski. Population fragmentation may reduce fertility to zero in Banksia goodiia: demonstration of the Allee effect. Oecologia, 94(3) (1993), 446-450.

[24] R. Lande. Risks of population extinction from demographic and environmental stochasticity and random catastrophes. Am. Nat., 142(6) (1993), 911-927.

[25] R. Lande. Extinction thresholds in demographic models of territorial populations. Am. Nat., 130(4) (1987), 624-635.

[26] J. G. Skellam. Random dispersal in theoretical populations. Biometrika, 38(1/2) (1951), 196-218.

[27] W. F. Laurance and E. Yensen. Predicting the impacts of edge effects in fragmented habitats. Biol. Conserv., 55(1) (1991), 77-92.

[28] M. Lewis, P. Kareiva. Allee dynamics and the spread of invading organisms. Theor. Popul. Biol., 43(2) (1993), $141-158$.

[29] A.Y. Morozov and B.L. Li. On the importance of dimensionality of space in models of space-mediated population persistence. Theor. Popul. Biol., 71(3) (2007), 278-289.

[30] D. Moser, H. G. Zechmeister, C. Plutzar, N. Sauberer, T. Wrbka, and G. Grabherr. Landscape patch shape complexity as an effective measure for plant species richness in rural landscapes. Landsc. Ecol., 17(7) (2002), 657-669.

[31] J. Murray. Mathematical Biology. Springer, 2002.

[32] S. V. Petrovskii and B. Li. Exactly Solvable Models of Biological Invasion. CRC Press, 2005.

[33] S. V. Petrovskii, B. Li. An exactly solvable model of population dynamics with density-dependent migrations and the Allee effect. Math. Biosci., 186(1) (2003), 79-91.

[34] B. J. Rathcke. Habitat fragmentation and plant pollinator. Curr. Sci. , 65(3) (1993), 273-277.

[35] N. Richter-Dyn and N. S. Goel. On the extinction of a colonizing species. Theor. Popul. Biol., 3(4) (1972), $406-433$.

[36] L. Ries, T. D. Sisk. A predictive model of edge effects. Ecology, 85(11) (2004), 2917-2926.

[37] D. W. Schemske, B. C. Husband, M. H. Ruckelshaus, C. Goodwillie, I. M. Parker, J. G. Bishop. Evaluating approaches to the conservation of rare and endangered plants. Ecology, 75(3) (1994), 584-606.

[38] C. Thies, T. Tscharntke. Landscape structure and biological control in agroecosystems. Science, 285 (5429) (1999), 893-895.

[39] V. Volpert, S. Petrovskii. Reaction-diffusion waves in biology. Phys. Life. Rev., 6(4) (2009), 267-310. 